ARTICLE

DOl: 10.1038/s41467-018-03164-5 OPEN

\title{
Physical basis of amyloid fibril polymorphism
}

\author{
William Close ${ }^{1}$, Matthias Neumann ${ }^{2}$, Andreas Schmidt ${ }^{1}$, Manuel Hora ${ }^{3}{ }^{3,4}$, Karthikeyan Annamalai ${ }^{1}$, \\ Matthias Schmidt', Bernd Reif ${ }^{3,4}$, Volker Schmidt ${ }^{2}$, Nikolaus Grigorieff ${ }^{5}$ \& Marcus Fändrich ${ }^{1}$
}

Polymorphism is a key feature of amyloid fibril structures but it remains challenging to explain these variations for a particular sample. Here, we report electron cryomicroscopy-based reconstructions from different fibril morphologies formed by a peptide fragment from an amyloidogenic immunoglobulin light chain. The observed fibril morphologies vary in the number and cross-sectional arrangement of a structurally conserved building block. A comparison with the theoretically possible constellations reveals the experimentally observed spectrum of fibril morphologies to be governed by opposing sets of forces that primarily arise from the $\beta$-sheet twist, as well as peptide-peptide interactions within the fibril cross-section. Our results provide a framework for rationalizing and predicting the structure and polymorphism of cross- $\beta$ fibrils, and suggest that a small number of physical parameters control the observed fibril architectures.

\footnotetext{
${ }^{1}$ Institute of Protein Biochemistry, Ulm University, 89081 Ulm, Germany. ${ }^{2}$ Institute of Stochastics, Ulm University, 89081 Ulm, Germany. ${ }^{3}$ Institute for Structural Biology, Helmholtz Zentrum München, 85764 Neuherberg, Germany. ${ }^{4}$ Munich Center for Integrated Protein Science (CIPS-M) at Department Chemie, Technische Universität München (TUM), Lichtenbergstr. 4, 85747 Garching, Germany. ${ }^{5}$ Janelia Research Campus, Howard Hughes Medical Institute, 19700 Helix Drive, Ashburn, VA 20147, USA. William Close, Matthias Neumann and Andreas Schmidt contributed equally to this work. Correspondence and requests for materials should be addressed to N.G. (email: niko@grigorieff.org) or to M.Fän. (email: marcus.faendrich@uni-ulm.de)
} 
A myloid fibrils are filamentous protein states that were originally identified in the context of systemic amyloidosis or neurodegenerative diseases such as Alzheimer's and Parkinson's ${ }^{1-3}$. More recently, however, amyloid-like fibrils have been suggested as novel devices in materials sciences, chemical engineering, and bionanotechnology ${ }^{4}$. Amyloid fibrils possess a unique structure that is characterized by a cross- $\beta$-sheet conformation in which $\beta$-strands run transversely to the main fiber axis and form an intermolecular network of hydrogen bonds ${ }^{1,5}$. They consist of one or multiple protofilaments $(\mathrm{PFs})^{6-11}$. They usually exhibit a width of $5-20 \mathrm{~nm}^{6,7,11-13}$, an overall polar topology $1,6,8-11,14$, a left-handed fibril supertwist $^{6-9,11,14}$, and a twofold helical symmetry ${ }^{6,8,9,11,14}$. A particular remarkable feature about amyloid fibril structures is their polymorphism.

Polymorphism refers to structural variations among different amyloid fibrils formed by a particular polypeptide chain, which can usually be observed with single-particle techniques when comparing the individual fibril structures in a sample 6 ,7,11-13,15. This intrasample polymorphism can be found irrespective of whether fibrils were formed in vitro ${ }^{6,7,11,15}$ or extracted from a patient or diseased animal ${ }^{12,13,16}$. Changing the conditions of fibril formation affects the spectrum of fibril morphologies that can be obtained within a test tube ${ }^{15,17}$, and different fibril morphologies may underlie the formation of different variants of amyloid diseases in vivo. Evidence in this regard has been provided for the aggregates formed by $A \beta$ peptide, prion protein, transthyretin, or serum amyloid A protein ${ }^{18-21}$.

Due to this polymorphism, predicting the formation of amyloid structures can be considered to be more complex than the conventional protein-folding problem, as the protein-folding reaction connects a given amino acid sequence with an essentially singular globular conformation ${ }^{22}$. In contrast, solutions to the "protein misfolding problem" are required to explain the polymorphic variations and the formation of multiple conformational end states by a polypeptide chain ${ }^{6,7,11-13,15,17,23}$. Previous analyses of polymorphic fibrils have revealed them to be linked with alterations in the number, orientation, or substructure of the PFs constructing different fibril polymorphs $s^{6,7,11,16,23,24}$ and have led to hypotheses concerning the possible modes of alternative packing of polypeptide chain ${ }^{25}$. However, it remains challenging to explain the specific structural assemblies seen within an experimental sample.
Using electron cryomicroscopy (cryo-EM), we have analyzed the polymorphism of fibrils formed by a peptide fragment from an immunoglobulin light chain causing systemic AL amyloidosis $^{8}$. This peptide, termed AL1, possesses a sequence (IGSNVVTWYQQL) that is devoid of charged amino acid side chains, confining all of its ionic groups to the peptide N- and Ctermini. For one of its fibril morphologies, termed hereafter morphology I, we previously obtained a cryo-EM reconstruction at sub-nm resolution ${ }^{8}$. This fibril encompassed six PFs, each consisting of a face-to-face packed pair of two cross- $\beta$ sheets. The fibril exhibited only two ways by which two PFs were packed within the mature fibril. One possibility involved a selfcomplementary packing of the lateral surfaces of two $\beta$-sheets that is mediated by inter-PF contacts between the hydrophobic and polar amino acid side chains. The other possibility involved electrostatic contacts between the complementary charged peptide $\mathrm{N}$ - and C-termini at the edges of the $\beta$-sheets in adjacent PFs. In this study, we extended this analysis to nine additional fibril morphologies formed by the AL1 peptide under the same conditions as morphology I. The obtained cryo-EM reconstructions were used to develop an algorithm to rationalize and predict the formation of specific fibril morphologies from this peptide.

\section{Results}

Cryo-EM reconstructions of different fibril morphologies. Analysis of the width of AL1 peptide fibrils with cryo-EM revealed a broad range of diameters from 8 to $22 \mathrm{~nm}$ (Supplementary Figure 1), demonstrating the variation of fibril structures in this sample, which we previously used to identify morphology $\mathrm{I}^{8}$. We now obtained reconstructions from nine additional fibril morphologies (II to X) that are present in this sample. For four reconstructions, we achieved resolutions of better than $1 \mathrm{~nm}$ (morphologies II to V) when estimated using the Fourier shell correlation (FSC) criterion at 0.143 (Supplementary Figure 2 and 3, Supplementary Table 1). The remaining fibrils were reconstructed at resolutions of $1.3-2.0 \mathrm{~nm}$ (Supplementary Table 1). Each fibril morphology presented a unique crosssectional architecture (Fig. 1b). The reconstructions of morphologies III, V, VI, and VIII-X remained essentially unaltered when twofold axial symmetry was imposed during refinement and reconstruction (Supplementary Figure 4), suggesting that this symmetry was present. In contrast, the reconstructions of morphologies II, IV, and VII were strongly altered when twofold

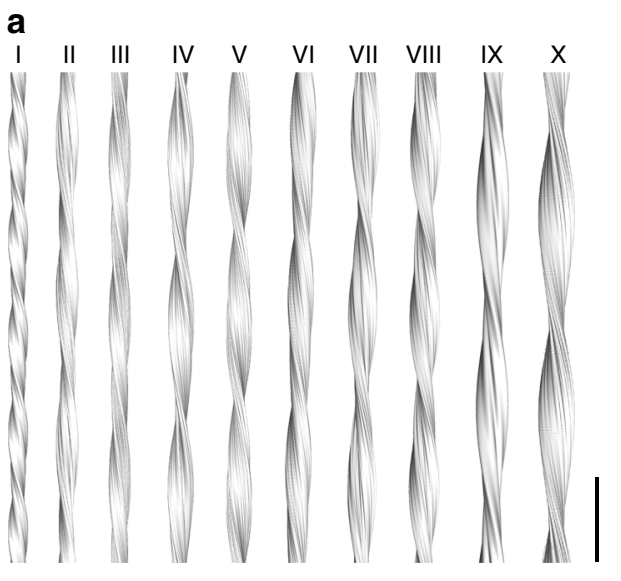

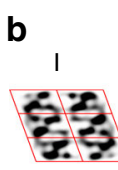
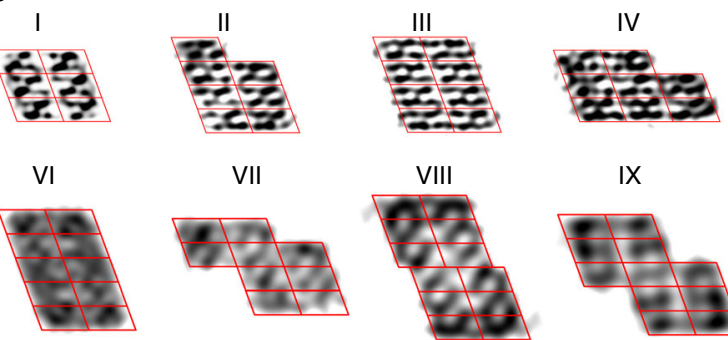

IX
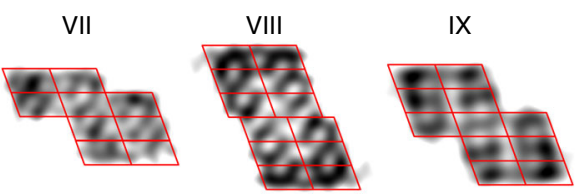

C
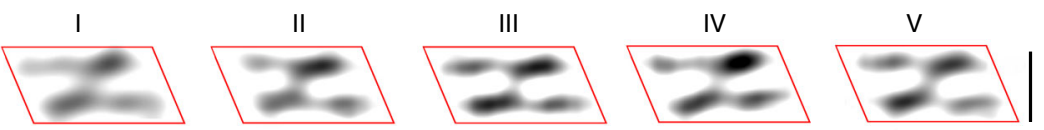

Fig. 1 Structural polymorphism of AL1 peptide fibrils. a Side view of reconstructions of fibril morphologies I-X (scale bar, $50 \mathrm{~nm}$ ). $\mathbf{b}$ Cross-sectional slices of the reconstructed densities superimposed with a lattice of parallelograms (red). Reconstructions of morphologies I-V were filtered to $10 \AA$ and morphologies $\mathrm{VI-X}$ to a resolution corresponding to their FSC values at 0.143 (scale bar, $5 \mathrm{~nm}$ ). c Aligned and averaged parallelograms of morphologies I-V sharing a quasi-twofold symmetry. The data from morphology I were included as published ${ }^{8}$ (scale bar, $2 \mathrm{~nm}$ ) 
axial symmetry was imposed, indicating that these filaments do not possess this symmetry (Supplementary Figure 4), and morphologies II, IV, and VII are shown here without an imposed symmetry. All fibrils were identified as polar by the imageprocessing software FREALIX ${ }^{26}$. We imposed a left-handed helical twist during reconstruction consistent with platinum side shadowing ${ }^{8}$. Each fibril morphology presented a unique crosssectional architecture (Fig. 1b) from which we could calculate the polar moment of inertia $I$ as a geometrical characteristic (Supplementary Methods). I represents a measure of the resistance of a fibril toward twisting and correlates, in the set of analyzed fibrils, with the pitch (Supplementary Figure 5). This finding relates to previous observations made with various amyloidogenic polypeptide chains, in which the pitch correlates with either $I$ or fibril width $6,11,15,27,28$. It indicates that the torsional features of the global fibril morphology, and thus the three-dimensional (3D) fibril structure, are determined by the two-dimensional (2D) architecture of the fibril cross section.

A conserved building block constructs the different fibrils. Analysis of the cross-sections of the various fibril morphologies identified a common building block that could be outlined by a parallelogram (Fig. 1b). A cross-correlation analysis of morphologies $\mathrm{I}-\mathrm{V}$ established the average dimensions of axes $a$ and $b$ of the parallelogram at $4.22 \pm 0.31 \mathrm{~nm}$ and $2.29 \pm 0.09 \mathrm{~nm}$. The angle $\gamma$ between the two axes was determined at $69.8 \pm 3.2^{\circ}$ (Supplementary Table 2). As each of these building blocks corresponded to the cross-section through a PF, the analyzed fibrils consisted of six to fourteen PFs, one of the highest PF numbers reported so far. The analyzed fibrils could be grouped into a "family tree" according to their increase in complexity (Supplementary Figure 6). Although finer details of the peptide conformation, such as the specific backbone dihedral angles or side chains, were not resolved at the current resolutions, each parallelogram enclosed two elongated density regions at a spacing distance of $\sim 11 \AA$. These densities were observed within fibril morphologies I-V (Fig. 1) and with lower confidence also within fibril morphologies VIII, IX, and X (Supplementary Figure 7).
Their size, density modulation, and quasi-twofold symmetry implied a face-to-face packed peptide dimer to underlie the fibril building blocks ${ }^{8}$.

Conservation of the interactions between the peptide dimers. There were primarily two ways by which two peptide dimers (or parallelograms) could be packed within a fibril cross-section. One possibility involved an in-register packing along axis $a$ of the parallelogram (long side interactions, LSIs) and the other, also inregister, along axis $b$ (short side interactions, SSIs). In-register LSIs and SSIs were shared by all 10 fibril morphologies, although fibril morphology VIII showed an additional off-register LSI in the fibril center (Supplementary Figure 7). LSIs were previously shown to arise from nonionic interactions between the exclusively hydrophobic and polar amino acid side chains, while SSIs involved electrostatic contacts between adjacent peptide $\mathrm{N}$ - and C-termini ${ }^{8}$. The number of LSIs per fibril tended to be greater than the number of SSIs. Some fibril morphologies show discernible gaps in the corners of their cross-section, corresponding to the size of a parallelogram. The parallelograms were only missing in the blunt corners of some of the cross-sections but never in the sharp corners, suggesting that unfilled sharp corners are structurally unfavorable.

Analysis of the protomer structure by ss-NMR. The structural conservation of the building blocks and that of the peptide conformation in different fibrils was supported by magic angle spinning (MAS) solid-state-NMR (ss-NMR) spectroscopy data obtained with uniformly ${ }^{13} \mathrm{C} /{ }^{15} \mathrm{~N}$-labeled AL1 peptide fibrils. Despite the clear polymorphism of the analyzed sample, the NMR measurements yielded well-resolved spectra (Fig. 2a) with methyl ${ }^{13} \mathrm{C}$ resonances exhibiting a line width of $\sim 130 \mathrm{~Hz}$ (0.33 p.p.m.). Most residues were associated with a single set of chemical shifts with the exception of Ser3 and Thr7, which resolved two sets of chemical shifts. However, the variations of these two residues did not discernibly affect the prediction of the peptide conformation by the program TALOS $+{ }^{29}$, which yielded essentially the same extended $\beta$-strand conformation with both sets of chemical shifts a

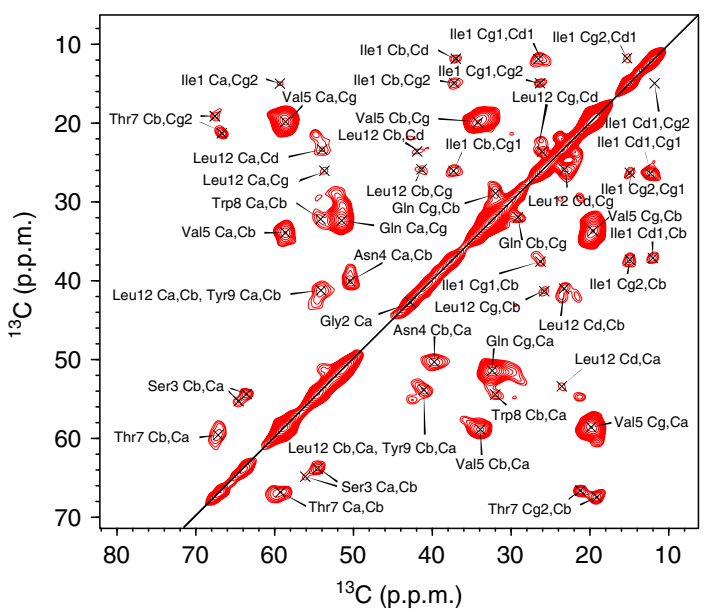

b

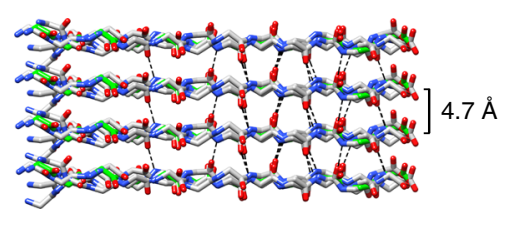

C

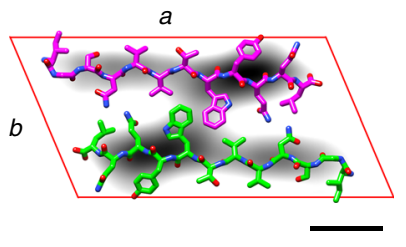

d

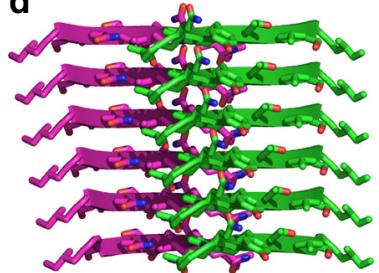
$a^{9}$

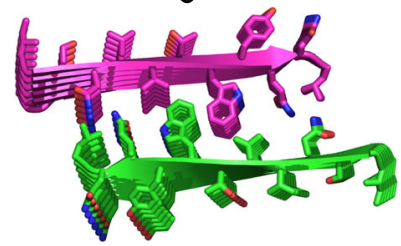

Fig. 2 Protomer conformation revealed by ss-NMR. a The $2 \mathrm{D}{ }^{13} \mathrm{C}-{ }^{13} \mathrm{C}$ proton-driven spin diffusion spectrum shows the cross-peaks between aliphatic

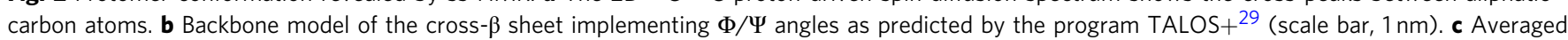
parallelograms of morphologies I-V (gray) superimposed with a peptide dimer (green, magenta) as obtained by NMR. The placement of the peptides followed the considerations described previously for morphology $I^{8}$ (scale bar, $1 \mathrm{~nm}$ ). d Side view and top view of a six-layer stack illustrating the structure of a PF. Side-chain conformations were not determined by experiments and represent only conformations that are compatible with the packing shown here 
a

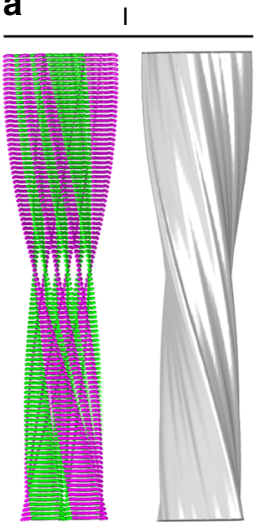

b

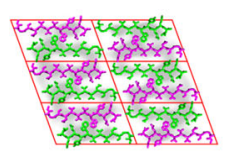

II

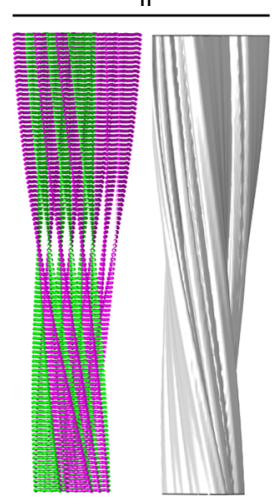

III

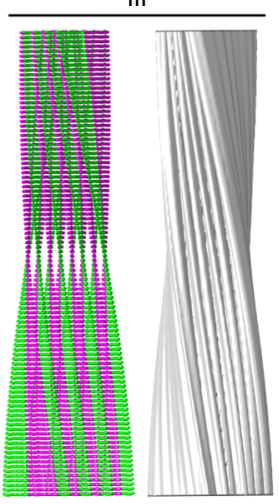

IV

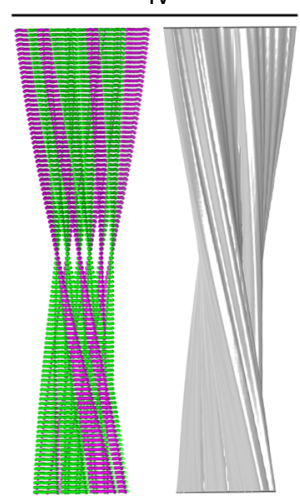

$\mathrm{V}$

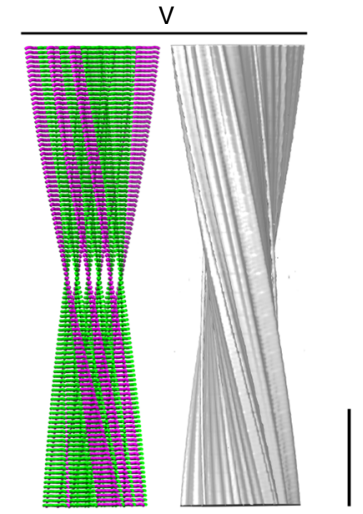

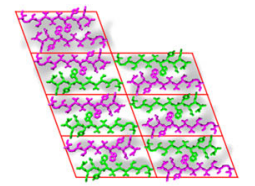
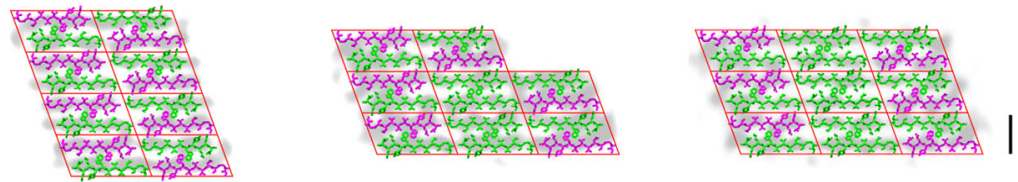

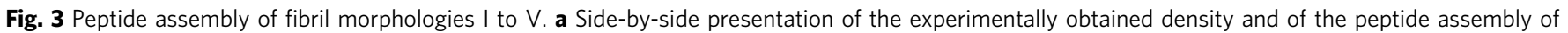

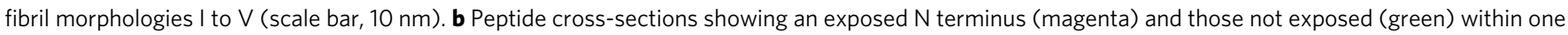
peptide layer, superimposed with reconstructions (gray) and filtered to $10 \AA$. Side-chain conformations were not determined by experiments and represent only conformations that are compatible with the packing shown here. The 3D map of morphology I was included as published ${ }^{8}$ (scale bar, 2 nm)

(Supplementary Figure 8c, d). The $\Phi / \Psi$-pairs occurred at the right side of the diagonal of the Ramachandran plot (Supplementary Figure $8 \mathrm{~b}$ ), indicating a left-handed $\beta$-sheet twist when viewed in the direction of the backbone hydrogen bonds ${ }^{30}$. This orientation of the twist is consistent with the orientation of the fibril supertwist as established previously by platinum side shadowing $^{8}$. A peptide model implementing the NMR-based $\Phi / \Psi$ constraints provided a good fit to the elongated densities enclosed by the parallelograms. Stacking multiple peptide dimers into a PF depicts a pair of two self-complementary cross- $\beta$ sheets (Fig. 2b-d) and the structural hierarchy of fibril morphologies I-V (Fig. 3).

Physical factors influencing fibril polymorphism. Having established that different fibril morphologies consisted of a common building block and that these fibrils show mainly two ways to pack the building blocks within the fibril cross-section, we wondered how many fibril morphologies (cross-sections) can be generated by a given number $n$ of building blocks or PFs? Furthermore, why are only some observed by experiments? To illustrate this problem, we found 120 possible arrangements encompassing $n=6$ building blocks with only one being observed by cryo-EM (Supplementary Figures 9, 10) and a total of 346,649 possible constellations for the entire range of $1 \leq n \leq 12$ (Supplementary Figure 11), eight of which were observed. As a next step, we considered the energetic contributions $E_{\mathrm{l}}, E_{\mathrm{s}}$, and $E_{\mathrm{e}}$ provided by a LSI, SSI, and an empty sharp corner to the fibril stability and combined them to define an arbitrary energy score $E$ for each fibril morphology as shown in Eq. (1) (for details please refer to the "Methods" section).

$$
E=E_{\mathrm{l}} n_{\mathrm{l}}+E_{\mathrm{s}} n_{\mathrm{s}}+E_{\mathrm{e}} n_{\mathrm{e}}+\alpha I^{2}
$$

In this equation, $n_{1}$ refers to the number of in-register LSIs, $n_{\mathrm{s}}$ to the number of SSIs, and $n_{\mathrm{e}}$ to the number of empty sharp corners. Additionally, we considered the possible effects arising from $I$, the polar moment of inertia, which we scaled relative to $E$ by the factor $\alpha$. The definition of $E$ followed the sign conventions of the Gibbs free energy $(\Delta G)$ in which a lower $E$ value implies a higher thermodynamic stability. We then computed the values of $n_{\mathrm{l}}, n_{\mathrm{s}}, n_{\mathrm{e}}$, and $I$ for all 346,649 fibril morphologies and split the resulting data set into two parts, one encompassing all fibril morphologies with an even number of PFs, and one encompassing all fibril morphologies with an odd PF number. Each subset was then evaluated with Eq. (1), termed hereafter as odd and even fits, to yield values for $E_{\mathrm{l}}, E_{\mathrm{s}}$, and $E_{\mathrm{e}}$ (Supplementary Table 4). These values were used to compute the stability of all fibril morphologies (Fig. 4, Supplementary Figure 12). This analysis possesses predictive power in those cases where the obtained values for $E_{\mathrm{l}}, E_{\mathrm{s}}$, and $E_{\mathrm{e}}$ were used to compute $E$ of fibrils from the other subset; that is, when odd-fit data were used to predict evennumbered fibrils and vice versa.

On the basis of this approach, we found that the experimentally observed fibril morphologies belong to the $1 \%$ most stable crosssectional arrangements for each respective $n$ value (Fig. 4c, Supplementary Figure 12b). Morphologies I, II, III, IV, V, and VI represented the most stable fibril architectures for $n=6,7,8,9$, and 10. Together with fibril morphology VII, they ranked within the 67 (even fit) or 49 (odd fit) most favorable fibril morphologies overall (Fig. 4, Supplementary Figure 12). There is an intermediate region of $n$ in which the formed fibrils could become particularly stable. This intermediate region was observed irrespective of whether we analyzed the lowest energy state at each $n$ value (Supplementary Figure 13a, b) or whether we counted the number of fibril morphologies below a certain threshold of $E$ (Supplementary Figure 13c, d). The intermediate region coincided with the population maximum when analyzing the fibril ensemble with mass-per-length (MPL) measurements (Supplementary Figure 13e).

The signs of $E_{s}$ and $E_{1}$ were found to be negative based on the fit and of $E_{e}$ to be positive. In other words, the fibril structures arose from opposing sets of the following forces: stabilizing forces associated with LSIs and SSIs, and unfavorable forces originating 
a

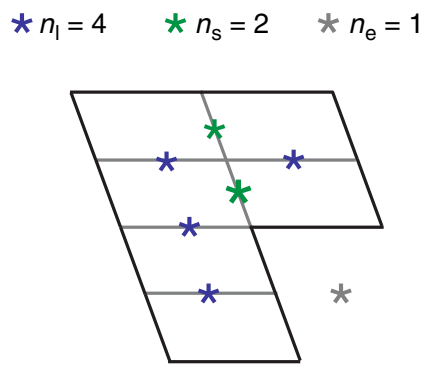

b

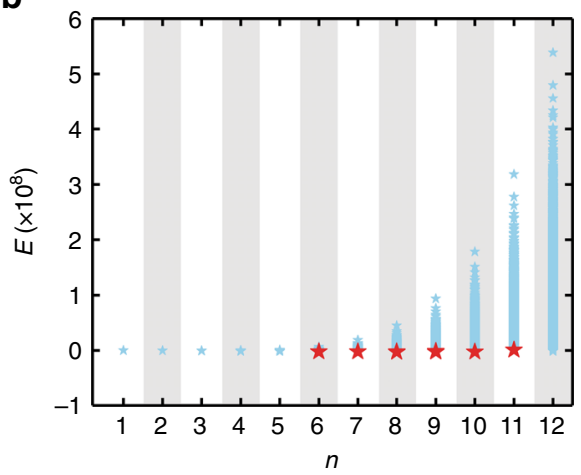

C

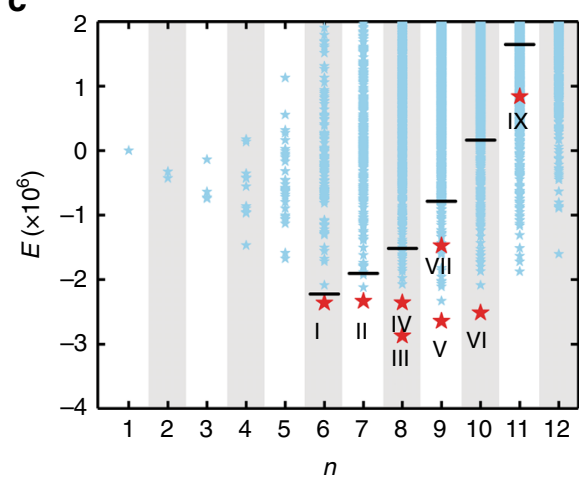

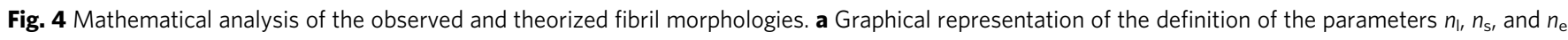
for a given fibril cross-section. $\mathbf{b}$ Plot of $E$ for all theorized fibril morphologies or cross-sections (blue) vs. $n$ as obtained by the even fit (gray columns). Hence, only white columns have predictive power for the even fit. Red symbols: experimentally observed fibril morphologies. c Close-up of $\mathbf{b}$. Black horizontal division markers show the $1 \%$ cutoff of the most stable morphologies for each $n$ value

from an unfilled sharp corner and $I$. The compromise between these opposing sets of forces was fibrils with relatively compact cross-sections (low $I$ values, high $\mathrm{n}_{\mathrm{l}}$, and $\mathrm{n}_{\mathrm{s}}$ values) (Supplementary Figure 12c), which is in accordance with the tightly knit nature of the fibril cross-sections seen by cryo-EM (Fig. 1). It is a consequence of our theory that the fibril width adopts a relatively narrow range of values, which we found by cryo-EM to range from 6 to $22 \mathrm{~nm}$ in AL1 peptide fibrils (Supplementary Figure 1). The presently developed theory might apply to other cross- $\beta$ fibril systems as indicated in Supplementary Figure 14 for the fibrils formed from a peptide fragment of transthyretin (TTR) 11.

\section{Discussion}

In this study, we have used cryo-EM to characterize the structural variations of AL1 peptide fibril morphologies. We found that different fibril morphologies arise from different combinations of similarly structured building blocks. The obtained cryo-EM data enabled us to analyze the formation of the observed fibril structural variants as compared to the theoretically possible alternative fibril arrangements. We found that a small number of physical parameters controls fibril architectural features in the experimental sample.

The simplifications applied in our analysis give rise to a number of limitations explaining the discrepancies between our theory and some sample properties. For example, we have reconstructed only ten fibril morphologies by cryo-EM, but additional fibril morphologies were identified in our cryo-EM images. Thus, other fibril configurations beyond the ones reconstructed here could be theorized to have a similar stability. Our analysis was further restricted to consider only in-register LSIs and SSIs, whereas morphology VIII encompasses additional off-register LSIs within the fibril core. In addition, the analyzed fibril morphologies were formed under a single set of conditions, suggesting that the AL1 peptide may be able to adopt fibril morphologies beyond the ones considered by our theory.

We ignored the peptide-peptide interactions along the main fibril axis and assumed $E_{\mathrm{l}}, E_{\mathrm{s}}$, and $E_{\mathrm{e}}$ to be independent from their exact radial and azimuthal positions in the twisted fibril helix. This assumption may break down progressively as the fibril width increases, limiting the confidence of our analysis at larger $n$ values. Equation (1) is also solely based on energy and does not consider kinetic contributions in defining the polymorphism of fibril morphologies ${ }^{31}$. As kinetic factors can be assumed to favor fibrils with a small number of PFs, which may nucleate and grow out faster than fibrils consisting of many more PFs, our analysis likely overestimates the formation of fibrils with large $n$ values.

Other limitations come from the fact that we cannot measure the stability of single morphologies experimentally nor derive a theoretical energy threshold to discriminate between possible and impossible fibril morphologies. Hence, it is difficult to compare our $E$ score with $\Delta G$ values obtained for specific fibril morphologies. However, we have noted that fibril morphologies VII and IX, which were rare in our sample and contributed only a few raw fibril images to our reconstructions (Supplementary Table 1), presented a low stability by our $E$ score. In contrast, morphology I did not result in being the most stable fibril morphology in both the odd and even fit, even though it was the most abundant morphology in solution. Ultimately, our analysis depends on the knowledge of the PF structure, which is difficult to predict for longer polypeptide chains, such as $A \beta$ peptide, $\alpha$-synuclein, or tau, which encompass non- $\beta$ segments or regions of conformational variability $1,3,9,16,32$.

Despite these limitations, our theory is able to explain the formation of the majority of the AL1 peptide fibrils observed by experiments. This ability implies that the incorporated physical properties are relevant for the formation of these cross- $\beta$ fibrils. We identified two opposing sets of forces to control the structure and formation of AL1 peptide fibrils, favorable forces arising from LSIs and SSIs, and unfavorable forces arising from unfilled sharp corners and $I$. At the molecular chemical level, these findings imply that the desire of the peptide to make intermolecular interactions within the cross-sectional plane, which promotes the lateral fibril growth and the formation of thick fibrils, is in competition with the twisting of the cross- $\beta$-sheet structure. As the $\beta$-sheet twist is counteracted by $I$, increasing the lateral extension of the fibril cross-section and the fibril thickness will make the formation of fibrils progressively unfavorable.

It is clear that several of the specific structural features considered by our quantitative analysis with Equation 1, such as the existence of LSIs and SSIs as well as empty sharp corners, may not exist in this form in other amyloid systems and specifically in those with a more complex fold of the polypeptide chain in the fibril. However, our theory incorporates a number of general structural features of amyloid fibrils. For example, a twisted fibril structure has been described for the amyloid fibrils formed from many polypeptide chains ${ }^{6-9,11,14,16,24}$, and there is, for many amyloid systems, evidence for a correlation between the fibril pitch and the fibril width or $I^{6,11,15,27,28}$. Moreover, the lateral fibril width of amyloid fibrils ranges frequently between 5 and 20 
$\mathrm{nm}^{6,7,11-13,16,32}$, similar to AL1 peptide fibrils (Supplementary Figure 1), and the formation of peptide-peptide interactions orthogonal to the direction of the backbone hydrogen bonds has been crucial in defining the steric zipper structures of amyloid fibril spines ${ }^{1,25}$. Moreover, Supplementary Figure 14 demonstrates that our theory can also be applied to rationalize the fibril structures formed from a peptide fragment of $\mathrm{TTR}^{11}$. Hence, it appears that the presently described theory of a competition between cross-sectional peptide-peptide interactions and fibril twist could be relevant for amyloid structures more generally.

Our observations provide a framework toward predicting amyloid structures, which has to consider the following three complexity levels: (i) the fold of the polypeptide chain, (ii) the packing of one or several polypeptide chains into a PF, and (iii) the arrangement of multiple PFs into a fibril. While previous studies primarily focused on the first two complexity levels ${ }^{33-36}$, our study addresses the arrangement of multiple PFs into a fibril. This analysis was greatly enabled by the structural simplicity of AL1 peptide and the fact that the PF-PF contacts occurred almost exclusively via in-register LSIs and SSIs. Therefore, it will be a task for future studies to improve our theory to predict the possible PF-PF interactions of longer polypeptide chains, such as in $\mathrm{A} \beta$ and tau fibrils, for which cryo-EM has recently provided evidence for alternative arrangements of similarly structured PFs ${ }^{6,16}$.

\section{Methods \\ Source of the peptide and conditions of fibril formation. Unlabeled and uni- formly ${ }^{13} \mathrm{C} /{ }^{15} \mathrm{~N}$ isotopically labeled variants of AL1 peptide were chemically syn- thesized at the Interdisziplinäres Zentrum für Klinische Forschung Leipzig, Core Unit Peptide-Technologien. Incubation of the peptide formed fibrils at a con- centration of $5 \mathrm{mg} / \mathrm{mL}$ in $50 \mathrm{mM}$ Tris at $\mathrm{pH} 8.0$ for a minimum of 3 days at room temperature $\left(20^{\circ} \mathrm{C}\right)$}

Transmission electron cryomicroscopy. A fibril solution $(14 \mu \mathrm{g} / \mathrm{mL})$ was applied onto glow-discharged C-flat holey carbon grids (CF 1.2/1.3-2 C) and after backside blotting, plunged into liquid ethane. Images were collected at $300 \mathrm{kV}$ in two sessions, initially with an FEI Falcon I direct electron detector (DED) mounted on an FEI F30 electron microscope at a total exposure of $\sim 20 \mathrm{e} / \AA^{2}$ and later with a K2 Summit (Gatan) DED mounted on an FEI Titan Krios electron microscope. The K2 Summit DED was operated in super-resolution mode to collect movies with 73 frames $(0.3 \mathrm{~s} /$ frame $)$ and a total exposure of $\sim 40 \mathrm{e} / \AA^{2}$.

Image processing. The movie frames collected on the K2 Summit were background corrected before motion correction was applied by aligning the frames with the program Unblur ${ }^{37}$. All frames were used in the final frame sums. Furthermore, the images collected on the Titan Krios were corrected for magnification distortion using the program mag_distortion_correct ${ }^{38}$. Fibril coordinates were selected using the program TigrisDisplay ${ }^{39}$. Fibrils were grouped according to their crossover length and width. The FREALIX helical image-processing software was used for 3D reconstruction. Axial symmetry of the fibrils was identified by comparison of asymmetric and twofold-symmetrized reconstructions. When both reconstructions gave similar results, a twofold axial symmetry was assumed, otherwise no axial symmetry was assumed (Supplementary Figure 4). Helical symmetry was imposed for all morphologies by using a helical rise calculated from the crossover distances and cross- $\beta$ repeat (Supplementary Table 1 ). The polarity of the analyzed fibrils was determined by FREALIX to ensure the correct alignment during the reconstruction.

MAS solid-state NMR spectroscopy. Packing of the MAS rotor was accomplished by sedimenting $12 \mathrm{mg}$ of fibrils at $21,000 \times g$ into the rotor, using a benchtop centrifuge. A 3.2-mm thin-wall $\mathrm{ZrO}_{2}$ rotor with vespel cap (CortecNet, Voisins Le Bretonneux, France) was employed with one house-made Teflon spacer $(1 \mathrm{~mm})$ at the bottom of the rotor. Proton-driven spin diffusion experiments ${ }^{40}$ with a mixing time of $50 \mathrm{~ms}$ were recorded using a $400 \mathrm{MHz}$ Bruker Avance III spectrometer (Bruker BioSpin, Karlsruhe). Sequential assignment was accomplished with $2 \mathrm{D}$ versions of NCACX and NCOCX experiments ${ }^{41}$, conducted using a 750$\mathrm{MHz}$ Bruker Avance III spectrometer (Bruker BioSpin, Karlsruhe). Carbon-carbon mixing was achieved by $50 \mathrm{~ms}$ of proton-driven spin diffusion. Nonuniform sampling (NUS) was employed to increase the sensitivity of the NCACX and NCOCX experiments ${ }^{42}$. Exponentially decaying sampling densities were used with $50 \%$ NUS sparsity and a $3-\mathrm{ms}$ time constant in the ${ }^{15} \mathrm{~N}$ dimension. Spectra were processed with the NUS plug-in of TopSpin 3.2 (Bruker BioSpin, Karlsruhe), using the compressed sensing option. For all measurements, the temperature of the probe was adjusted to $0{ }^{\circ} \mathrm{C}$. All experiments used $100 \mathrm{kHz}$ of ${ }^{1} \mathrm{H}$ decoupling using SPINAL- $64^{43}$ and $10 \mathrm{kHz}$ of MAS spinning. Spectra were analyzed with CcpNmr Analysis 2.4.0 $0^{44}$. Secondary structure analysis was accomplished with TALOS $+^{29}$ based on the ${ }^{13} \mathrm{Ca},{ }^{13} \mathrm{C} \beta,{ }^{13} \mathrm{C}$ backbone carbonyl, and ${ }^{15} \mathrm{~N}$ backbone amide chemical shifts (Supplementary Table 3$)^{29}$. The analyzed fibrils were formed under conditions identical to our cryo-EM samples. They were structurally indistinguishable from those used for reconstruction as judged by negative-staining TEM (Supplementary Figure 8a).

Theoretical diversity of fibril cross-sections. To generate all possible constellations of fibril cross-sections consisting of $1 \leq n \leq 12$ parallelograms, we used an iterative approach, in which we set out from the fibril consisting of a single parallelogram $(n=1)$, that was deposited within a list $L_{1}$. Assuming only inregister LSIs and SSIs, we then added one adjacent parallelogram to the existing cross-section to generate all possible cross-sections containing two parallelograms $(n=2)$. These cross-sections were temporarily stored within a second list $L_{2}$ (Supplementary Figure 9a). Nonredundant cross-sections were added to $L_{1}$ and the remaining cross-sections in $L_{2}$ were deleted. This cycle was repeated, always adding one parallelogram after the other until the value $n=12$ was reached.

Data availability. The fibril structural data that support the findings of this study have been deposited and made available in the Electron Microscopy Data Bank. Morphology I was previously made available with the accession no. EMD-3128. The newly generated fibril structures from morphology II to X are also publicly available (accession numbers for morphology II: EMD-3986, III: EMD-3987, IV: EMD-3988, V: EMD-3989, VI: EMD-3990, VII: EMD-3991, VIII: EMD-3992, IX: EMD-3993, and X: EMD-3994). Other data are available from the corresponding authors upon reasonable request.

Received: 25 July 2017 Accepted: 24 January 2018

Published online: 16 February 2018

\section{References}

1. Riek, R. \& Eisenberg, D. S. The activities of amyloids from a structural perspective. Nature 539, 227-235 (2016).

2. Sipe, J. D. et al. Amyloid fibril proteins and amyloidosis: Chemical identification and clinical classification International Society of Amyloidosis 2016 Nomenclature Guidelines. Amyloid 23, 209-213 (2016).

3. Chiti, F. \& Dobson, C. M. Protein misfolding, functional amyloid, and human disease. Annu. Rev. Biochem. 75, 333-366 (2006).

4. Cherny, I. \& Gazit, E. Amyloids: not only pathological agents but also ordered nanomaterials. Angew. Chem. Int. Ed .Engl. 47, $4062-4069$ (2008).

5. Sunde, $\mathrm{M}$. et al. Common core structure of amyloid fibrils by synchrotron $\mathrm{x}$ ray diffraction. J. Mol. Biol. 273, 729-739 (1997).

6. Meinhardt, J., Sachse, C., Hortschansky, P., Grigorieff, N. \& Fändrich, M. A $\beta$ (1-40) fibril polymorphism implies diverse interaction patterns in amyloid fibrils. J. Mol. Biol. 386, 869-877 (2009).

7. Jiménez, J. L. et al. The protofilament structure of insulin amyloid fibrils. Proc Natl Acad. Sci. USA 99, 9196-9201 (2002).

8. Schmidt, A., Annamalai, K., Schmidt, M., Grigorieff, N. \& Fändrich, M. CryoEM reveals the steric zipper structure of a light chain-derived amyloid fibril. Proc. Natl Acad. Sci. USA 113, 6200-6205 (2016).

9. Schmidt, M. et al. Peptide dimer structure in an $A \beta(1-42)$ fibril visualized with cryo-EM. Proc. Natl Acad. Sci. USA 112, 11858-11863 (2015).

10. Fändrich, M., Schmidt, M. \& Grigorieff, N. Recent progress in understanding Alzheimer's $\beta$-amyloid structures. Trends Biochem. Sci. 36, 338-345 (2011).

11. Fitzpatrick, A. W. et al. Atomic structure and hierarchical assembly of a cross$\beta$ amyloid fibril. Proc. Natl Acad. Sci. USA 110, 5468-5473 (2013).

12. Annamalai, K. et al. Common fibril structures imply systemically conserved protein misfolding pathways in vivo. Angew. Chem. Int. Ed. Engl. 56, 7510-7514 (2017)

13. Annamalai, K. et al. Polymorphism of amyloid fibrils in vivo. Angew. Chem. Int. Ed. Engl. 55, 4822-4825 (2016).

14. Sachse, C., Grigorieff, N. \& Fändrich, M. Nanoscale flexibility parameters of alzheimer amyloid fibrils determined by electron cryo-microscopy. Angew. Chem. 49, 1321-1323 (2010).

15. Klement, K. Effect of different salt ions on the propensity of aggregation and on the structure of Alzheimer's A $\beta(1-40)$ amyloid fibrils. J. Mol. Biol. 373, 1321-1333 (2007)

16. Fitzpatrick, A. W. P. et al. Cryo-EM structures of tau filaments from Alzheimer's disease. Nature 547, 185-190 (2017).

17. Kodali, R., Williams, A. D., Chemuru, S. \& Wetzel, R. A $\beta(1-40)$ forms five distinct amyloid structures whose $\beta$-sheet contents and fibril stabilities are correlated. J. Mol. Biol. 401, 503-517 (2010). 
18. Collinge, J., Sidle, K. C. L., Meads, J., Ironside, J. \& Hill, A. F. Molecular analysis of prion strain variation and the aetiology of 'new variant' CJD. Nature 383, 685-690 (1996).

19. Heilbronner, G. et al. Seeded strain-like transmission of $\beta$-amyloid morphotypes in APP transgenic mice. Embo. Rep. 14, 1017-1022 (2013).

20. Bergström, J. et al. Amyloid deposits in transthyretin-derived amyloidosis: cleaved transthyretin is associated with distinct amyloid morphology. $J$. Pathol. 206, 224-232 (2005).

21. Westermark, G. T., Fändrich, M. \& Westermark, P. A. AAmyloidosis: Pathogenesis and target therapy. Annu. Rev. Pathol. Mech. Dis. 10, 321-344 (2014).

22. Anfinsen, C. B. Principles that govern the folding of protein chains. Science 181, 223-230 (1973).

23. Fändrich, M., Meinhardt, J. \& Grigorieff, N. Structural polymorphism of Alzheimer A $\beta$ and other amyloid fibrils. Prion 3, 89-93 (2009).

24. Schmidt, $M$. et al. Comparison of Alzheimer $A \beta(1-40)$ and $A \beta(1-42)$ amyloid fibrils reveals similar protofilament structures. Proc. Natl Acad. Sci. USA 106, 19813-19818 (2009).

25. Wiltzius, J. J. W. et al. Molecular mechanisms for protein-encoded inheritance. Nat. Struct. Mol. Biol. 16, 973-978 (2009).

26. Rohou, A. \& Grigorieff, N. Frealix: Model-based refinement of helical filament structures from electron micrographs. J. Struct. Biol. 186, 234-244 (2014).

27. Bauer, H. H. et al. Architecture and polymorphism of fibrillar supramolecular assemblies produced by in vitro aggregation of human calcitonin. J. Struct. Biol. 115, 1-15 (1995).

28. Abe, H. \& Nakanishi, H. Effect of $\mathrm{pH}$ on the aggregate formation of a nonamyloid component (1-13). J. Pept. Sci. 9, 177-186 (2003).

29. Shen, Y., Delaglio, F., Cornilescu, G. \& Bax, A. TALOS+: A hybrid method for predicting protein backbone torsion angles from NMR chemical shifts. J. Biomol. Nmr. 44, 213-223 (2009).

30. Chothia, C. Conformation of twisted $\beta$-pleated sheets in proteins. J. Mol. Biol. 75, 295-302 (1973).

31. Pellarin, R. \& Caflisch, A. Interpreting the aggregation kinetics of amyloid peptides. J. Mol. Biol. 360, 882-892 (2006).

32. Tuttle, M. D. et al. Solid-state NMR structure of a pathogenic fibril of fulllength human a-synuclein. Nat. Struct. Mol. Biol. 23, 409-415 (2016).

33. Thompson, M. J. et al. The 3D profile method for identifying fibril-forming segments of proteins. Proc. Natl Acad. Sci. USA 103, 4074-4078 (2005).

34. Ahmed, A. B. \& Kajava, A. V. Breaking the amyloidogenicity code: methods to predict amyloids from amino acid sequence. FEBS Lett. 587, 1089-1095 (2013).

35. Bryan, A. W. Jr et al. BETASCAN: probable b-amyloids identified by pairwise probabilistic analysis. PLoS Comput. Biol. 5, 1-11 (2009).

36. O'Donnell, C. W. et al. A method for probing the mutational landscape of amyloid structure. Bioinformatics 27, 34-42 (2011).

37. Grant, T. \& Grigorieff, N. Measuring the optimal exposure for single particle cryo-EM using a $2.6 \AA$ reconstruction of rotavirus VP6. eLife 4, 1-19 (2015).

38. Grant, T. \& Grigorieff, N. Automatic estimation and correction of anisotropic magnification distortion in electron microscopes. J. Struct. Biol. 192, 204-208 (2015).

39. Grant, T. \& Grigorieff, N. TigrisDisplay, Grigorieff lab(Janelia Research Campus, Virginia, USA) http://tigris.sourceforge.net Tigris (2009).

40. Grommek, A., Meier, B. H. \& Ernst, M. Distance information from protondriven spin diffusion under MAS. Chem. Phys. Lett. 427, 404-409 (2006).

41. Pauli, J., Baldus, M., van Rossum, B., de Groot, H. \& Oschkinat, H. Backbone and side-chain ${ }^{13} \mathrm{C}$ and ${ }^{15} \mathrm{~N}$ signal assignments of the $\alpha$-spectrin $\mathrm{SH} 3$ domain by magic angle spinning solid-state NMR at 17.6 Tesla. Chem. Bio. Chem. 2, 272-281 (2001).

42. Palmer, M. R. et al. Sensitivity of nonuniform sampling NMR. J. Phys. Chem. B. 119, 6502-6515 (2015).

43. Fung, B. M., Khitrin, A. K. \& Ermolaev, K. An improved broadband decoupling sequence for liquid crystals and solids. Journal of Magnetic Resonance. J. Magn. Reson. 142, 97-101 (2000).

44. Vranken, W. F. et al. The CCPN data model for NMR spectroscopy: development of a software pipeline Proteins. Struct., Funct., Bioinforma. 59, 687-696 (2005).

\section{Acknowledgements}

This work is supported by grants from the German Federal Ministry of Education and Research (GERAMY - German consortium for systemic light chain (AL) amyloidosis, 01GM1107) and the National Institutes of Health (P01 GM62580, awarded to N.G). W.C. acknowledges a stipend from the Landesgraduiertenförderungsgesetzes BadenWürttemberg.

\section{Author contributions}

W.C., A.S., N.G., and M.F. wrote the paper. W.C., M.N., A.S., and M.H. conducted the research. W.C., M.N., A.S., M.H., M.S., B.R., V.S., N.G., and M.F. analyzed the data. M. N., K.A., and V.S. provided materials and analysis tools. M.S., B.R., V.S., N.G., and M.F. designed the research. All authors discussed the results and commented on the manuscript.

\section{Additional information}

Supplementary Information accompanies this paper at https://doi.org/10.1038/s41467018-03164-5.

Competing interests: The authors declare no competing financial interests.

Reprints and permission information is available online at http://npg.nature.com/ reprintsandpermissions/

Publisher's note: Springer Nature remains neutral with regard to jurisdictional claims in published maps and institutional affiliations.

Open Access This article is licensed under a Creative Commons Attribution 4.0 International License, which permits use, sharing, adaptation, distribution and reproduction in any medium or format, as long as you give appropriate credit to the original author(s) and the source, provide a link to the Creative Commons license, and indicate if changes were made. The images or other third party material in this article are included in the article's Creative Commons license, unless indicated otherwise in a credit line to the material. If material is not included in the article's Creative Commons license and your intended use is not permitted by statutory regulation or exceeds the permitted use, you will need to obtain permission directly from the copyright holder. To view a copy of this license, visit http://creativecommons.org/ licenses/by/4.0/

(c) The Author(s) 2018 